\title{
Development and first experimental tests of Faraday cup array
}

\author{
J. Prokůpek, ${ }^{1,2,3, a)}$ J. Kaufman, ${ }^{2}$ D. Margarone, ${ }^{1}$ M. Krůs, ${ }^{1,2,3}$ A. Velyhan, ${ }^{1}$ J. Krása, ${ }^{1}$ \\ T. Burris-Mog, ${ }^{4}$ S. Busold, ${ }^{5}$ O. Deppert, ${ }^{5}$ T. E. Cowan, ${ }^{4,6}$ and G. Korn ${ }^{1}$ \\ ${ }^{1}$ Institute of Physics of the AS CR, v. v. i., ELI-Beamlines Project, Na Slovance 2, 18221 Prague 8, \\ Czech Republic \\ ${ }^{2}$ Faculty of Nuclear Sciences and Physical Engineering, Czech Technical University in Prague, Břehová 7 , \\ 11519 Prague 1, Czech Republic \\ ${ }^{3}$ Institute of Plasma Physics of the AS CR, v. v. i./PALS Centre, Za Slovankou 3, 18200 Prague 8, \\ Czech Republic \\ ${ }^{4}$ Helmholtz-Zentrum Dresden-Rossendorf (HZDR), Bautzner Landstraße 400, D-01328 Dresden, Germany \\ ${ }^{5}$ Technische Universität Darmstadt (TUD), Schlossgartenstraße 9, D-64289 Darmstadt, Germany \\ ${ }^{6}$ Technische Universität Dresden, D-01069 Dresden, Germany
}

(Received 29 October 2013; accepted 14 December 2013; published online 9 January 2014)

\begin{abstract}
A new type of Faraday cup, capable of detecting high energy charged particles produced in a high intensity laser-matter interaction environment, has recently been developed and demonstrated as a real-time detector based on the time-of-flight technique. An array of these Faraday cups was designed and constructed to cover different observation angles with respect to the target normal direction. Thus, it allows reconstruction of the spatial distribution of ion current density in the subcritical plasma region and the ability to visualise its time evolution through time-of-flight measurements, which cannot be achieved with standard laser optical interferometry. This is a unique method for two-dimensional visualisation of ion currents from laser-generated plasmas. A technical description of the new type of Faraday cup is introduced along with an ad hoc data analysis procedure. Experimental results obtained during campaigns at the Petawatt High-Energy Laser for Heavy Ion Experiments (GSI, Darmstadt) and at the Prague Asterix Laser System (AS CR) are presented. Advantages and limitations of the used diagnostic system are discussed. (O) 2014 AIP Publishing LLC. [http://dx.doi.org/10.1063/1.4859496]
\end{abstract}

\section{INTRODUCTION}

A variety of novel Faraday cup detectors have been recently introduced. ${ }^{1-9}$ Different designs enable various measurement techniques: ion collectors for ion guns, ${ }^{2}$ micro-scale Faraday cups in electron microscopy ${ }^{3}$ Faraday cups reducing secondary electron emission in plasma focus experiments, ${ }^{4}$ Faraday cups with fast time response for measuring electron beams of traveling wave tube guns. ${ }^{5}$ Generally speaking, Faraday cups are used for various types of experimental measurements: ion mass spectrometry; ${ }^{6}$ beam current measurement of an ion source where electromagnetic noise is associated $;{ }^{7}$ alpha particle detection in tokamaks ${ }^{8}$ ion detection from laser-plasma based experiments. ${ }^{9}$ The main technique used at Prague Asterix Laser System (PALS) ${ }^{10}$ for ion current measurements with Faraday cups, as well as other detectors, is the time-of-flight (TOF) method. ${ }^{9,11-13}$

By using several Faraday cups in array geometry, it is possible to observe the expansion of a laser produced plasma from different detection angles at the same time in order to reconstruct the ion current angular distribution. Faraday cup arrays have already been tested for ion diagnostics in various environments. ${ }^{14-16}$

In this paper, a new type of miniature Faraday cup is presented and technically described in Sec. II. This type of

a) Author to whom correspondence should be addressed. Electronic mail: prokupek@fzu.cz. detector, used as an ion collector in array geometry and in TOF configuration, is capable of detecting high currents coming from the laser-generated plasma particles onto the detector. The main difficulty of such measurements is that the detector is in an environment where large Electromagnetic Pulse (EMP) exists, especially when the detector is placed only few tens of centimetres from the target. The EMP has a broad spectrum ( $\mathrm{MHz}$ up to $\mathrm{GHz}$ ) of wavelengths resonating inside the vacuum vessel and influencing all electronic devices. ${ }^{17}$ For this reason the temporal length of the EMP affects the TOF signal by generating a large noise signal on the oscilloscope which interferes with any detector signal.

A data analysis method is developed and described in detail in Sec. III. The experimental setup is described in Sec. IV together with the results from the Faraday cup array at PALS, which are mainly used to reconstruct the time evolution of plasma ion products. The first test of the Faraday cup array in the frame of fs-laser ion production was carried out at the Petawatt High-Energy Laser for Heavy Ion EXperiments (PHELIX $)^{18}$ laser facility in GSI, Darmstadt, whereas another experimental campaign was carried out at the sub-nanosecond PALS facility, Prague. Besides this new type of Faraday cup, different diagnostics were used during the experimental campaign: a pinhole camera, an X-ray streak camera, a visible interferometer, a Thomson parabola spectrometer, as well as other TOF detectors (silicon carbide detectors, scintillators, and different types of Faraday cups). 


\section{TECHNICAL DESCRIPTION OF THE DEVELOPED FARADAY CUP}

Usually, traditional ion collectors have been employed in laser driven ion acceleration experiments carried out at nanosecond laser facilities. They consist of a flat copper electrode, in front of which a conductive grid is placed. A given bias voltage is applied to the electrode, whereas the grid is grounded. ${ }^{19}$ This configuration yields a final signal on a fast oscilloscope (typically $1 \mathrm{GHz}$ ), which is composed of an ion TOF spectrum itself and a fast signal due to secondary electrons emitted from the metallic electrode and the grid (caused by photoelectric mechanism of the incoming plasma extreme ultraviolet radiation). Such a signal can last for several tens of nanoseconds (up to $100 \mathrm{~ns}$ ) and partially overlaps with the rising edge of the ion signal. Usually, in order to achieve a reasonable time (velocity) resolution, such an ion collector is placed far from the source. Hence, it has another disadvantage due to the fact that it has to be connected to a long tube which is mounted onto a vacuum flange where the possibilities of investigating the ion emission at different detection angles are limited. An additional problem is the above mentioned EMP associated with the interaction of high intensity lasers with solid targets. For those reasons, a new type of Faraday cup has been developed where both the geometry and the electronic components have been optimized with the following goals: (i) flexibility of placing them at different angles inside the target chamber; (ii) reducing the secondary electron emission and EMP signal; (iii) maintaining a high time resolution in TOF configuration even at short distances from the source.

The new device consists of two concentric shaped cylindrical electrodes made of copper with a teflon insulator between them. The outer cylinder is a shielding electrode connected to the ground. Diameters of the inner and the outer electrode were designed as small as possible but large enough to allow for physical construction in a workshop without any need for a special machining sequence. The inner cylinder is made with a hole along the axis but not penetrating through the electrode completely. The dimensions of the Faraday cups electrodes are sketched in Figure 1. This configuration of the Faraday cup with the hole in the inner electrode ensures that the detector will have properties of an emission-less ion col-

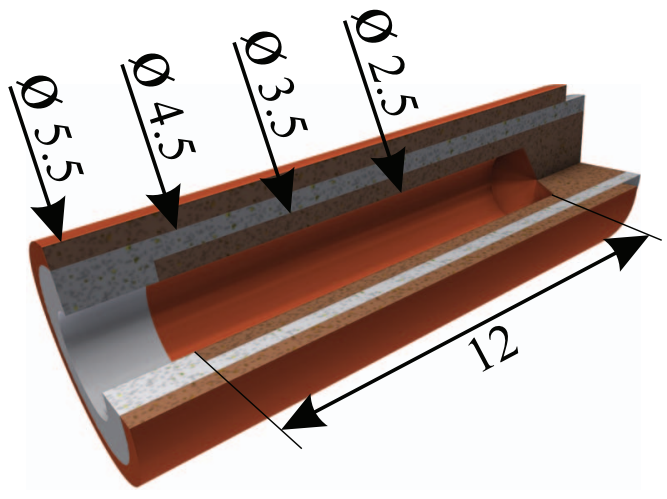

FIG. 1. Schematic drawing of the new type of Faraday cup. The inner and the outer copper electrodes with teflon insulator between them are depicted with dimensions given in $\mathrm{mm}$. The length of the whole device is $16 \mathrm{~mm}$ excluding the length of the standard cable connectors. lector because the effect of the emission of electrons induced by impinging ions is strongly suppressed.

To characterize properties of the new developed Faraday cup, one can assume it as a coaxial line transmitting the current signal given by the impinging charged particles. The important characteristic is the impedance defining the signal transmission characteristics from the detector to the coaxial cable and further to the oscilloscope; the impedance of the detector is $10.55 \Omega$. To apply the voltage on the Faraday cup, the signal transfer must be interrupted with a capacitor, separating the Faraday cup from the oscilloscope. For this reason, only high frequency signals can pass through and be registered.

\section{DATA ANALYSIS PROCEDURE}

Determination of TOF spectrum is the measurement of a time-resolved voltage amplitude induced by charged particles impinging the Faraday cup. The signal is registered at the oscilloscope (Tektronix DPO 7104 with sample rate of 5 GS/s was used at PHELIX, whereas Tektronix DPO 4104 with sample rate of $5 \mathrm{GS} / \mathrm{s}$ was used at PALS). As it can be seen in Figures 2(a) and 2(b), the first part of both signals is an interference made by the EMP, serving also as a trigger.
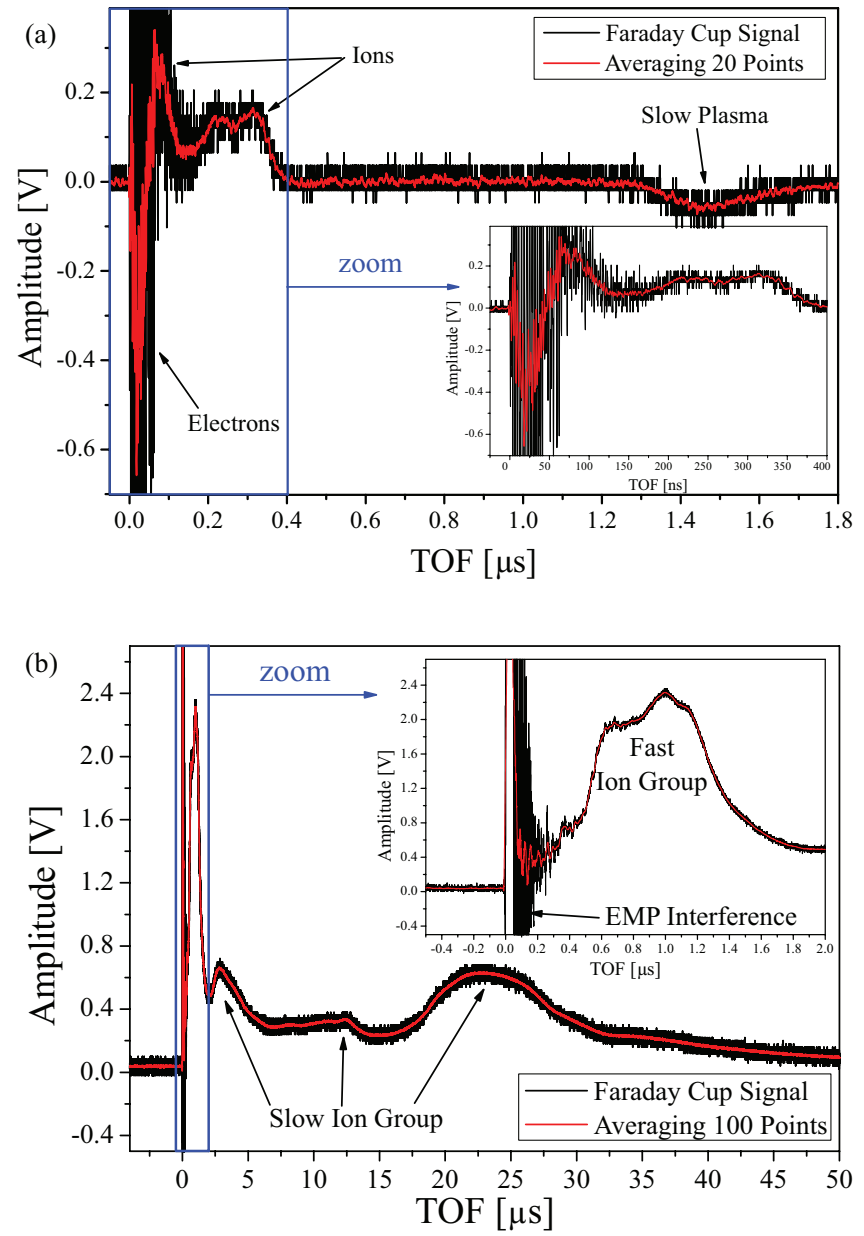

FIG. 2. Typical spectrum measured by the new type of Faraday cup (a) at PHELIX laser facility (TOF at $20 \mathrm{~cm}$ distance) and (b) at PALS facility (TOF at $50 \mathrm{~cm}$ distance). 
Using the given target-detector distance, it is possible to assign each point of the TOF spectra to a certain velocity value, and thus, transforming the TOF distribution into a velocity distribution.

The next step is to transform a time-resolved ion current $j(L, t)$ (i.e., time-of-flight spectrum of expanding ions) observed at a distance $L$ from the target into a distance-offlight dependence of the ion current $j(x, \tau)$ for a chosen value of the time-of-flight $\tau$ which is based on a similarity relationship of currents of ions having "frozen" charge state due to high rarefaction of ion density, ${ }^{20}$

$$
j(x, \tau) \cdot x^{3}=j(L, t) \cdot L^{3},
$$

where $x \tau=L t$. Thus, every velocity point calculated from the TOF signal is then assigned a certain space position. The current density is recalculated for all positions taking into account the original detector position and its solid angle. The current density $j$ at each position $L$ is determined by the relationship,

$$
j(L, t)=C \cdot \frac{U_{0}}{R S},
$$

where $U_{0}$ is the response of the Faraday cup in volts induced by collected ions, $R$ is the circuit resistance, $S$ is the detecting area of the Faraday cup, and $C$ is the correction to the transmission of the signal in the circuit and to a relative calibration. A correction factor for signal transmission and attenuation was used in the PALS experiment. By this way, every distance point is assigned a current density value. Using the known angle $\phi$ of the detector, $x$ and $y$ coordinates can be recalculated, whereas $z$ is associated with the current density value. This is then projected into a $2 \mathrm{D}$ plot, represented by colour contours, as it was described in Ref. 21. The data interpolation between each two neighbouring detectors is made in order to finalize the $2 \mathrm{D}$ plot.

If a grounded grid is applied in front of the Faraday cup, the secondary electron emission from the grid would have to be taken into account giving an additional signal recorded together with the TOF signal of the ions. Secondary electron emission experiments were done in the past, ${ }^{22}$ thus it is possible to calculate necessary corrections.

\section{EXPERIMENTAL RESULTS}

Faraday cups have been tested in two different laser facilities (PHELIX sub-picosecond and PALS sub-nanosecond) bringing them into two different conditions of operation. In the PHELIX experiment the Faraday cups were placed behind the target at a distance of about $20 \mathrm{~cm}$ and $60 \mathrm{~cm}$ to observe forwardly accelerated ions. They were located close to the target normal (distributed from target normal up to an angle of $25^{\circ}$ ). The laser with a central wavelength of $1.053 \mu \mathrm{m}$, pulse energy of about $100 \mathrm{~J}$ (before compression), and pulse duration of 500 fs (FWHM) was focused by a $45^{\circ}$ off-axis parabolic mirror onto a $10 \mu \mathrm{m}$ gold foil. The peak intensity in focus was about $1.4 \times 10^{19} \mathrm{~W} \mathrm{~cm}^{-2}$. At the PALS experiments the Faraday cup array was placed in front of the target (backward direction of the laser beam) and their distance varied from $40 \mathrm{~cm}$ to $50 \mathrm{~cm}$ from the target. One of the Faraday
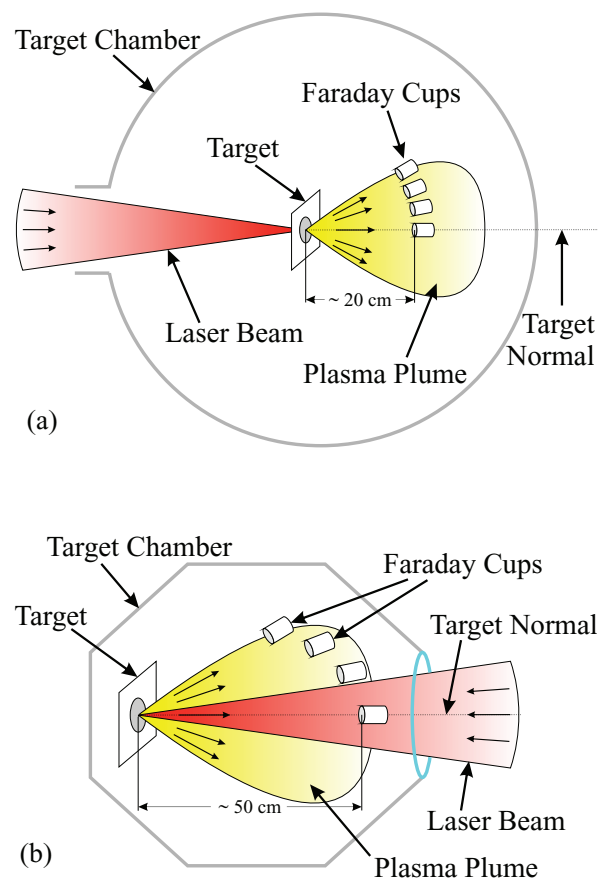

FIG. 3. Sketch of the experimental setup at PALS.

cups was placed in the target normal (laser axis) direction, whereas the rest were at angles between $30^{\circ}$ and $70^{\circ}$ with respect to the target normal (the laser beam incidence angle was $0^{\circ}$ ).

A typical detected signal from the Faraday cup is shown in Figure 2(a) at PHELIX and in Figure 2(b) at PALS. In both signals the first part (blue frame) represents the EMP noise lasting from tens to hundreds of nanoseconds (depending on the pulse length of the laser and the shielding capabilities of the detector). Moreover, the EMP noise level is in principle different when using two different laser peak intensities on target. The noise is followed by a fast ion group and then a slow one. To extract the 2D information, one needs to spread the detectors along a wide range of angles. This setup was arranged at the PALS experiment and the results reported below refer to it. The layout of the PALS experiment is shown in Figure 3.

The Faraday cup signals at PALS were recorded in numerous laser shots. The laser beam at its fundamental frequency of $1.315 \mu \mathrm{m}$, pulse energy of about $600 \mathrm{~J}$ and pulse duration of 300 ps (FWHM) was focused by an aspheric lens onto a double layer planar target. The foil was $2.5 \mu \mathrm{m}$ polyethylene terephthalate (PET) with a $50 \mathrm{~nm}$ aluminium substrate on one side. This target geometry was chosen to generate a simple ion current consisting of protons and carbon ions from the PET part of the target keeping relatively high EMP due to the presence of $\mathrm{Al}$ substrate. The peak intensity in focus was about $4.4 \times 10^{16} \mathrm{~W} \mathrm{~cm}^{-2}$. The spatial ion current density distribution is shown in Figure 4. This two-dimensional map of ion currents was determined from time-resolved signals of Faraday cups with the use of relationship (1) for a time of $222 \mathrm{~ns}$ after the laser-target interaction. The fast ion group expands along the target normal, whereas no fast ions are present at large angles. The white area close 


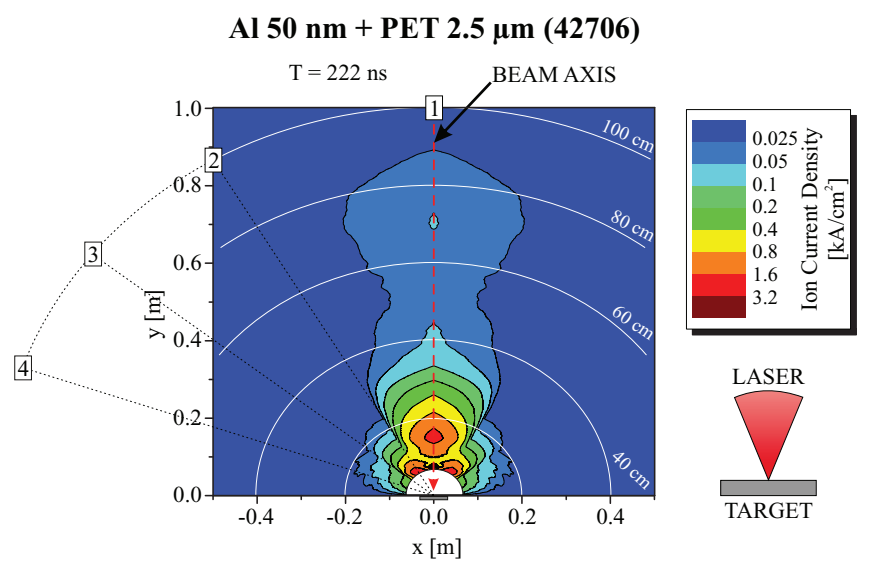

FIG. 4. Spatial distribution of ion current density using double layer target with $\mathrm{Al}$ on the front side and PET on the rear side irradiated with $516 \mathrm{~J}$ of laser energy.

to the target (see in Figure 4) represents the region of early plasma expansion where the relationship (1) is not valid due to several different plasma processes. ${ }^{23}$ Thus, the ion current density, measured far from the target (plasma free expansion region), is possibly underestimated. A cylindrical symmetry is assumed to visualise the $2 \mathrm{D}$ plot with the target normal in the middle. This symmetry is also assumed for evaluation of the plasma electron density from visible interferometry although a declination of the plasma plume expansion from the target surface normal can be expected. ${ }^{24}$ However, in other experiments, where the laser beam impacts with nonzero angle, the ions are emitted in non-normal direction. ${ }^{20}$

The result of interferometric measurements, covering an area in front of the target, reported in Figure 5 shows the plasma electron density distribution taken 500 ps after the main pulse hits the target. The interferometer uses part of the laser pulse at third harmonics $(438 \mathrm{~nm})$. It is possible to see the formation of a dense region along the target normal. This is supposed to correspond to the fast ion generation registered by the Faraday cup. Comparing the results in Figures 4 and 5, one can recognize similar structures of plasma formation, especially along the target normal (the fast ion signal

\section{Al 50 nm + PET $2.5 \mu \mathrm{m}(42706)$}

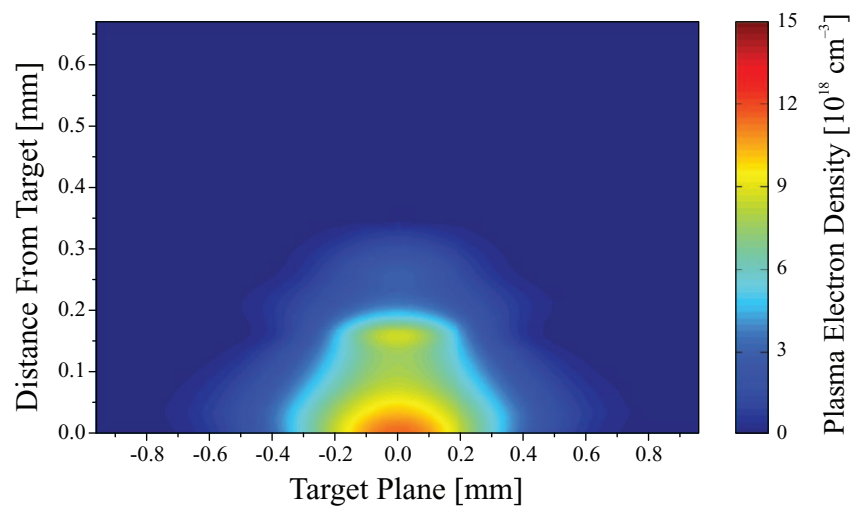

FIG. 5. Plasma electron density distribution from interferometry with target irradiated with $516 \mathrm{~J}$ of laser energy, $500 \mathrm{ps}$ after the main pulse arrival.

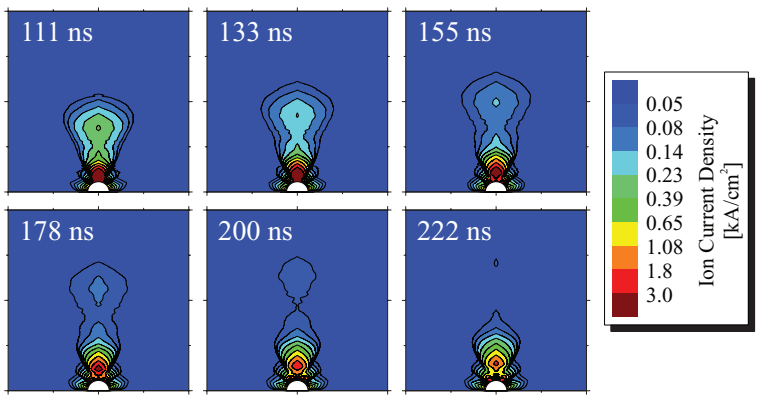

FIG. 6. Time evolution of plasma from shot shown in Figure 4. The ion current density scale is the same for all time steps.

in case of the Faraday cup and the region with higher electron density in case of the visible interferometry).

Figure 4 clearly demonstrates the presence of fast particles in the target normal direction. By choosing various times and applying the analysis procedure described in Sec. III on each time separately, one obtains a time evolution of the plasma plume. In Figure 6, six consecutive time steps (22 ns per each step) visualizing the time evolution of the plasma expansion are shown. For each time step, the ion current density scale is kept constant. The current density drops in each consecutive step as relationship (1) predicts. First, the time stretching of the signal due to the different particle velocities leads to fewer particles hitting the detector during the same time interval. Second, as the solid angle of observation decreases with increasing distance, ${ }^{25}$ the detected charge decreases because fewer particles hit the detector on the same unit of detectors area. For this reason, the current density is decreasing when the time increases. We note these statements are valid under the assumption that the charge separation is conserved, ${ }^{23}$ i.e., no neutralization mechanism occurs in plasma.

\section{SUMMARY AND CONCLUSION}

First experiments with the new type of Faraday cups showed promising features of the detectors. A configuration, where several detectors are placed at different angles, allows measuring the space distribution of current density of plasma ion components, similarly as it was done for ion charge density. ${ }^{20}$ The angular resolution of the obtained 2D contour plot of the ion current density is given by the total number of employed detectors available and the relative angular distance between each two neighbouring detectors. The main limitation in comparison with standard optical interferometry is, as previously mentioned, the low number of detectors and, as a consequence, the inaccuracy of data interpolation. The advantage, on the other hand, is the possibility to follow only the plasma ion emission (interferometry is more connected to the plasma electron emission and is limited to the undercritical density region) and to be able to measure very high ion currents (only limited by the attenuation required for oscilloscope protection, and the corresponding dynamic range). Another advantage of using Faraday cups is the possibility to determine the time evolution by changing the evaluation time (shown in Figure 6) as discussed above. Utilizing this method, 
it is possible to obtain a snapshot of the ion current and ion charge density distributions at any chosen time (except short times where the signal is hidden in the blank region), or to create a video sequence representing the time evolution of the ion current density in single shot experiments, which can often result in a better visualization of the plasma expansion relatively far from the target, which can be useful when particle detectors or optics have to be placed at a given distance from the source.

A further development of the new type of Faraday cups should focus on a better shielding of the detector from EMP interference. The proper building of a Faraday cage around the detector as well as shielding of the signal cables and the oscilloscope itself is a crucial point in order to be able to detect fast particles typically hidden in the EMP noise. In our experiment at PALS the fastest protons have energies exceeding $2 \mathrm{MeV}$ according to the Thomson parabola spectrometer measurement. In the used Faraday cup the TOF signal of those protons should appear at about $20 \mathrm{~ns}$, which overlaps with the $100 \mathrm{~ns}$ strong EMP interference. Decreasing the level of interference by proper shielding would cause the fastest part of the ion signal to emerge from the noise. After further development and experiments, one should verify that the technique of using Faraday cups for proton and ion radiography is also worthy for fast particles, applicable at petawatt-class laser that are becoming more widely used.

\section{ACKNOWLEDGMENTS}

This work benefitted from the support of the Czech Science Foundation (Project No. P205/11/1165), the Czech Republic's Ministry of Education, Youth and Sports to the ELI-Beamlines (CZ.1.05/1.1.00/02.0061), LaserGen (CZ.1.07/2.3.00/20.0087), LaserZdroj (CZ.1.07/2.3.00/ 20.0279) and PALS RI (LM2010014) projects, the Academy of Sciences of the Czech Republic (M100101210), the CHARPAC Work Package of the Laserlab-Europe III project (Grant No. 284464).

Authors would also like to thank the staff members at the PHELIX laser facility in GSI and laser and experimental personnel at the PALS facility.

${ }^{1}$ J. Hu and J. L. Rovey, "Faraday cup with nanosecond response and adjustable impedance for fast electron beam characterization," Rev. Sci. Instrum. 82, 073504 (2011).

${ }^{2}$ G. Hars and G. Dobos, "Development of analytically capable time-of-flight mass spectrometer with continuous ion introduction," Rev. Sci. Instrum. 81, 033101 (2010)

${ }^{3}$ F. Tang, X. Wang, L. Zhang and Z. Yan, "Study on simulation and experiment of array micro Faraday cup ion detector for FAIMS," Sci. Chin Technol. Sci. 53, 3225-3231 (2010).

${ }^{4}$ G. H. Roshani, M. Habibi, and M. Sohrabi, "An improved design of Faraday cup detector to reduce the escape of secondary electrons in plasma focus device by COMSOL," Vacuum 86, 250-253 (2011).

${ }^{5}$ Y.-X. Wei, M.-G. Huang, S.-Q. Liu, J.-Y. Liu, B.-L. Hao, C.-H. Du, and P.-K. Liu, "Faraday cup for measuring the electron beams of TWT guns," Vacuum 86, 2109-2113 (2012).

${ }^{6} \mathrm{~K}$. L. Busch, "Detecting ions in mass spectrometers with the Faraday cup," Spectroscopy 26, 12-18 (2011).

${ }^{7}$ M. R. B. Ghadikolaee and E. T. Ghadikolaee, "Design of a new Faraday cup to measure the beam current of an ion source with residual gas," J. Fusion Energy 31, 569-572 (2012).
${ }^{8}$ F. E. Cecil, V. Kiptily, D. S. Darrow, A. Murari, and J.-E. Contributors, "Status of the JET Faraday cup lost alpha particle diagnostic KA-2," in Proceedings of the 12th IAEA Technical Meeting on Energetic Particles in Magnetic Confinement Systems/5th IAEA Technical Meeting on Theory of Plasma Wave Instabilities, Austin, TX, 05-11 September 2011 [Nucl. Fusion 52, 094022 (2012)].

${ }^{9}$ D. Margarone, J. Krasa, L. Giuffrida, A. Picciotto, L. Torrisi, T. Nowak, P. Musumeci, A. Velyhan, J. Prokupek, L. Laska, T. Mocek, J. Ullschmied, and B. Rus, "Full characterization of laser-accelerated ion beams using Faraday cup, silicon carbide, and single-crystal diamond detectors," J. Appl. Phys. 109, 103302 (2011).

${ }^{10}$ K. Jungwirth, A. Cejnarova, L. Juha, B. Kralikova, J. Krasa, E. Krousky, P. Krupickova, L. Laska, K. Masek, T. Mocek, M. Pfeifer, A. Prag, O. Renner, K. Rohlena, B. Rus, J. Skala, P. Straka, and J. Ullschmied, "The Prague Asterix Laser System," in Proceedings of the 42nd Annual Meeting of the Division of Plasma Physics of the American-Physical-Society/10th International Congress on Plasma Physics, Quebec City, Canada, 23-27 October 2000 [Phys. Plasmas 8, 2495-2501 (2001)].

${ }^{11}$ A. Picciotto, L. Torrisi, S. Gammino, A. Mezzasalma, F. Caridi, D. Margarone, L. Ando, J. Krasa, L. Laska, and J. Wolowski, "Temperature measurements in plasmas generated by using lasers at different intensities," in Proceedings of the 2nd International Workshop on Production of Intense Beams of Highly Charged Ions and Pulse Plasma Laser Ablation, Giardini Naxos, Italy, 08-11 June 2005 [Radiat. Eff. Defects Solids 160, 705-713 (2005)].

${ }^{12}$ D. Margarone, J. Krasa, L. Laska, A. Velyhan, T. Mocek, J. Prokupek, E. Krousky, M. Pfeifer, S. Gammino, L. Torrisi, J. Ullschmied, and B. Rus, "Measurements of the highest acceleration gradient for ions produced with a long laser pulse," in Proceedings of the 13th International Conference on Ion Sources (ICIS'09), Gatlinburg, TN, 20-25 September 2009 [Rev. Sci. Instrum. 81, 02A506 (2010)].

${ }^{13}$ J. Prokůpek, D. Margarone, J. Hřebíček, M. Krůs, A. Velyhan, J. Pšikal, M. Pfeifer, T. Mocek, J. Krása, J. Ullschmied, K. Jungwirth, G. Korn, and B. Rus, "Pilot experiment on proton acceleration using the $25 \mathrm{TW}$ femtosecond Ti:Sapphire laser system at PALS," Nucl. Instrum. Methods Phys. Res. A 690, 48-52 (2012).

${ }^{14}$ R. Qindeel, N. Bidin, Z. Ibrahim, Y. M. Daud, and Nur-Shahidah, "Angular distribution of $\mathrm{Cu}$ ions by Nd:YAG laser using Faraday cups," Curr. Nanosci. 6, 315-319 (2010).

${ }^{15}$ J. Lorincik, M. B. Denton, R. P. Sperline, E. T. Young, and P. Williams, "Testing of a micro Faraday cup array for ion detection in SIMS," Anal. Lett. 44, 1050-1057 (2011).

${ }^{16}$ D. S. Darrow, F. E. Cecil, V. Kiptily, K. Fullard, A. Horton, A. Murari, and J. E. Contributors, "Observation of alpha particle loss from JET plasmas during ion cyclotron resonance frequency heating using a thin foil Faraday cup detector array," in Proceedings of the 18th Topical Conference on High-Temperature Plasma Diagnostics, Wildwood, NJ, 16-20 May 2010 [Rev. Sci. Instrum. 81, 10D330 (2010)].

${ }^{17}$ C. G. Brown, Jr., J. Ayers, B. Felker, W. Ferguson, J. P. Holder, S. R. Nagel, K. W. Piston, N. Simanovskaia, A. L. Throop, M. Chung, and T. Hilsabeck, "Assessment and mitigation of diagnostic-generated electromagnetic interference at the National Ignition Facility," in Proceedings of the 19th Topical Conference on High-Temperature Plasma Diagnostics, Monterey, CA, 06-10 May 2012 [Rev. Sci. Instrum. 83, 10D729 (2012)].

${ }^{18}$ V. Bagnoud, B. Aurand, A. Blazevic, S. Borneis, C. Bruske, B. Ecker, U. Eisenbarth, J. Fils, A. Frank, E. Gaul, S. Goette, C. Haefner, T. Hahn, K. Harres, H. M. Heuck, D. Hochhaus, D. H. H. Hoffmann, D. Javorkova, H. J. Kluge, T. Kuehl, S. Kunzer, M. Kreutz, T. Merz-Mantwill, P. Neumayer, E. Onkels, D. Reemts, O. Rosmej, M. Roth, T. Stoehlker, A. Tauschwitz, B. Zielbauer, D. Zimmer, and K. Witte, "Commissioning and early experiments of the PHELIX facility," Appl. Phys. B 100, 137-150 (2010).

${ }^{19}$ E. Woryna, P. Parys, J. Wolowski, and W. Mroz, "Corpuscular diagnostics and processing methods applied in investigations of laser-produced plasma as a source of highly ionized ions," Laser Part. Beams 14, 293-321 (1996).

${ }^{20}$ J. Krása, P. Parys, L. Velardi, A. Velyhan, L. Ryć, D. Delle Side, and V. Nassisi, "Time-of-flight spectra for mapping of charge density of ions produced by laser," Laser and Particle Beams, (2013) (published online).

${ }^{21}$ A. Kasperczuk, T. Pisarczyk, T. Chodukowski, Z. Kalinowska, P. Parys, O. Renner, S. Y. Gus'kov, N. N. Demchenko, J. Ullschmied, E. Krousky, M. Pfeifer, K. Rohlena, and J. Skala, "Laser-produced aluminum plasma expansion inside a plastic plasma envelope," Phys. Plasmas 19, 092106 (2012). 
${ }^{22}$ B. Svensson and G. Holmen, "Electron-emission from aluminum and copper under molecular-hydrogen-ion bombardment," Phys. Rev. B 25, 30563062 (1982).

${ }^{23}$ A. Lorusso, J. Krasa, K. Rohlena, V. Nassisi, F. Belloni, and D. Doria, "Charge losses in expanding plasma created by an XeCl laser," Appl. Phys. Lett. 86, 081501 (2005).
${ }^{24} \mathrm{~A}$. Thum-Jager and K. Rohr, "Angular emission distributions of neutrals and ions in laser ablated particle beams," J. Phys. D 32, 2827-2831 (1999).

${ }^{25}$ J. Krása, A. Lorusso, V. Nassisi, L. Velardi, and A. Velyhan, "Revealing of hydrodynamic and electrostatic factors in the center-of-mass velocity of an expanding plasma generated by pulsed laser ablation," Laser Part. Beams 29, 113-119 (2011). 